\title{
BMJ Open Study protocol for a randomised controlled trial evaluating the clinical effect of antibiotic prophylaxis in children with recurrent respiratory tract infections: the Approach study
}

\author{
Daphne Peeters (D) , , Nan van Geloven, ${ }^{2}$ Loes E Visser, ${ }^{3,4}$ Debby Bogaert,, ${ }^{5,6}$ \\ Annemarie M C van Rossum, ${ }^{7}$ Gertjan J A Driessen, ${ }^{1,8}$ Lilly M Verhagen (D) 6,9,10
}

To cite: Peeters D, van Geloven N, Visser LE, et al. Study protocol for a randomised controlled trial evaluating the clinical effect of antibiotic prophylaxis in children with recurrent respiratory tract infections: the Approach study. BMJ Open 2021;11:e044505. doi:10.1136/ bmjopen-2020-044505

- Prepublication history and additional supplemental material for this paper are available online. To view these files, please visit the journal online (http://dx.doi.org/10.1136/ bmjopen-2020-044505)

GJAD and LMV contributed equally.

Received 07 September 2020 Accepted 02 June 2021

Check for updates

(C) Author(s) (or their employer(s)) 2021. Re-use permitted under CC BY-NC. No commercial re-use. See rights and permissions. Published by BMJ.

For numbered affiliations see end of article.

Correspondence to

Lilly M Verhagen;

L.M.Verhagen-3@umcutrecht.nl

\section{ABSTRACT}

Introduction Respiratory tract infections (RTIs) affect children all over the world and are associated with significant morbidity and mortality. In particular, recurrent RTIs cause a high burden of disease and lead to frequent doctor visits. Children with recurrent RTIs generally have no significant alterations or deficits in systemic immunity. In an attempt to treat the assumed bacterial component involved, they are often treated with prolonged courses of prophylactic antibiotics taken on a daily basis. Despite its common use, there is no evidence that this is beneficial. Studies assessing the clinical effectiveness of antibiotic prophylaxis as well as potential adverse effects and antibiotic resistance development, are therefore urgently needed.

Methods and analysis We present a protocol for a randomised double-blind placebo-controlled trial comparing co-trimoxazole with placebo treatment in children with recurrent RTIs. A total of 158 children (aged 6 months -10 years) with recurrent RTIs without significant comorbidity will be enrolled from a minimum of 10 Dutch hospitals. One group receives co-trimoxazole $18 \mathrm{mg} / \mathrm{kg}$ two times per day $(36 \mathrm{mg} / \mathrm{kg} /$ day) and the other group receives a placebo two times per day for a period of 3 months. The main objective is to determine whether antibiotic prophylaxis is more effective than placebo to prevent/reduce respiratory symptoms in children with recurrent RTIs. Respiratory symptoms will be scored by parents on a daily basis in both study arms by the use of a mobile phone application. Our primary outcome will be the number of days with at least two respiratory symptoms during the treatment.

Ethics and dissemination Ethics approval was obtained from the Medical Ethics Research Committee Zuidwest Holland/LDD. A manuscript with the study results will be submitted to a peer-reviewed journal. All participants will be informed about the study results. The results of the study will inform clinical guidelines regarding the prophylactic treatment of children with recurrent RTIs.

Trial registration number NL7044.

\section{Strengths and limitations of this study}

- Apart from studies focusing exclusively on otitis media, this is the first randomised controlled trial that examines whether co-trimoxazole prophylaxis is effective to prevent/reduce symptoms in children with recurrent respiratory tract infections (RTIs).

- We will examine the clinical response to prophylactic antibiotic treatment both during the period in which treatment is taken and also in the 3 months following that period, because of an extended follow-up duration of 6 months in total.

- We will examine predictors of treatment effect, such as clinical characteristics, microbiota parameters and immunological characteristics.

- All children receive the same dose per $\mathrm{kg}$ bodyweight of co-trimoxazole and this study does not measure pharmacokinetic parameters. Therefore, we will not be able to determine the most optimal dosage of co-trimoxazole when prescribed for a prophylactic indication in children with recurrent RTIs.

- We will not enrol children with underlying chronic illnesses, such as cardiorespiratory or neuromuscular conditions, immune deficiency and congenital abnormalities, so the results of our study cannot be extrapolated to these patient groups.

\section{INTRODUCTION}

Lack of evidence-based guidelines for a common clinical problem

Young children (up to 2 years of age) experience symptoms of a respiratory tract infection (RTI) for a median of 44 days per year. The median number of infectious episodes is almost double in children with recurrent RTIs when compared with healthy peers. ${ }^{1}$ Even in the absence of high-risk conditions such as major immune deficiencies or congenital malformations, some children develop many more RTIs than their peers. ${ }^{2}$ Recurrent RTIs in children are among the leading reasons for 
primary care consultations and for referral to paediatricians and ear, nose and throat (ENT) specialists. ${ }^{3}$ In developed countries, recurrent RTIs, defined as a minimum of six to eight episodes per year, affect $15 \%-20 \%$ of children under 5 years of age. ${ }^{4}$ Most children suffer from recurrent RTIs of the upper airways, but in approximately $10 \%-30 \%$, the lower respiratory tract is also affected. ${ }^{5}$ Compared with their healthy peers, children with recurrent RTIs visit the outpatient clinic more often and they frequently need hospitalisation. In addition, these children are treated more frequently with medication, for example, inhaled bronchodilators and corticosteroids, as well as antibiotics. ${ }^{1}$ The high disease burden can also lead to failure to thrive and developmental delays in children, as well as parental productivity losses associated with children's illness and absenteeism from work, leading to high costs for the community. ${ }^{167}$ Lower RTIs in childhood can also lead to pulmonary sequelae such as bronchiectasis and irreversible lung damage, ${ }^{8-10}$ putting children with recurrent RTIs even more at risk for long-term damage. In our experience, children who visit the outpatient clinics because of recurrent RTIs are generally $\leq 10$ years of age and most are treated on a 'trial and error' base because there is no international consensus about the best (prophylactic) treatment for children with recurrent RTIs. Previous studies suggest that antibiotic prophylaxis, treatment with the immunomodulator OM-85, active immunisation and/or parental education on risk factors (passive smoking) may be at least of some benefit. ${ }^{411-14}$ In general, antibiotics are frequently used for the treatment of acute RTIs in children. In an attempt to treat the assumed bacterial component involved in recurrent RTIs, prolonged antibiotic regimens are often prescribed. Previous studies in children with recurrent acute otitis media showed that antibiotic prophylaxis prevented one and a half episode for every 12 months of treatment per child. ${ }^{15}$ Studies examining the clinical effectiveness of antibiotic prophylaxis in children suffering from recurrent RTIs are scarce and mainly focus on high risk groups with recurrent lower RTIs. ${ }^{11}{ }^{16-21}$ No studies have been performed on the clinical effectiveness of antibiotic prophylaxis in otherwise healthy children who suffer from recurrent RTIs.

The antibiotic regimen that is commonly prescribed in children with recurrent RTIs is trimethoprim/sulfamethoxazole (co-trimoxazole). Both trimethoprim and sulfamethoxazole are bacteriostatic if used alone. Combining trimethoprim and sulfamethoxazole elicits a synergistic effect and makes the antibiotic regimen bactericidal. Co-trimoxazole is a fixed antibiotic combination of trimethoprim and sulfamethoxazole (1:5) which covers most Gram-positive and Gram-negative potential pathogens as well as Pneumocystis jiroveci. In addition, several studies suggest that co-trimoxazole has an immunomodulatory effect. ${ }^{22-25}$ The combination of antimicrobial and immunomodulatory properties could provide an additional beneficial effect in the prevention or reduction of RTIs.
In this study, we will compare the clinical effectiveness of co-trimoxazole prophylaxis with placebo in children with recurrent RTIs.

\section{Role of microbiota in respiratory infection and disease}

Prolonged antibiotic treatment is of major concern, since antimicrobial resistance (AMR) development increases with duration of the antimicrobial course. ${ }^{26}$ Probably, the most important route of AMR gene selection in humans is antibiotic-induced changes to our protective microbial communities, also called the microbiota. ${ }^{27}$ Whereas microbial disturbances elicited by antibiotic treatment in adults are mostly temporary, exposure to antibiotic treatment early in life may have a lasting impact on the composition of the microbiota leading to permanent replacement by resistant organisms. ${ }^{28}{ }^{29}$ While the microbiota of the gastrointestinal tract has been studied most extensively, ${ }^{3031}$ the human nasopharynx is considered the niche from which both upper and lower RTIs originate and resistance can also emerge in commensals or pathogens colonising this body site. ${ }^{32}$ During the past decade, high-throughput pipelines have become available to also characterise the complete nasopharyngeal microbiota. ${ }^{33}$

In this study, we will examine short-term and long-term effects of co-trimoxazole prophylaxis on the microbiota composition and antibiotic resistance in children who suffer from recurrent RTIs.

\section{METHODS AND ANALYSIS Objectives and study parameters}

Primarily, we aim to investigate the clinical efficacy of co-trimoxazole prophylaxis in Dutch children (aged 6 months up to 10 years) with recurrent RTIs. Children will be randomised to co-trimoxazole or placebo for a treatment period of 3 months, since this is the treatment period after which a beneficial effect was achieved in children with recurrent acute or chronic suppurative otitis media. $^{15}$

\section{Primary objective}

To determine whether 3 months of prophylactic treatment with co-trimoxazole causes a reduction in the number of days a child experiences at least two RTI symptoms in children aged 6 months to $\leq 10$ years with recurrent RTIs, when compared with placebo.

\section{Secondary objectives}

1. To determine whether co-trimoxazole prophylactic therapy reduces:

- Time to resolution of symptoms.

- The severity of symptoms defined by the number and type of different symptoms.

- Use of analgesics/antipyretics.

- Use of antibiotic treatment courses.

- Absenteeism from day care or school and/or parental absenteeism from work.

- Alterations in nutritional status. 
Table 1 Inclusion and exclusion criteria

$\begin{array}{ll}\text { Inclusion criteria } & \text { Presenting to one of the participating clinics. } \\ & \text { Age } 6 \text { months-10 years. } \\ \text { Exclusion criteria } & \text { Suffering from recurrent respiratory tract infections (RTIs).* } \\ & \text { Current prophylactic antibiotic use or prophylactic antibiotic use during the } \\ \text { previous month. } & \text { Underlying immune deficiency other than for lgA or lgG subclasses. } \\ & \text { Congenital abnormalities (including but not limited to cleft palate, neuromuscular } \\ & \text { or cardiac disorders and syndromes). } \\ & \text { Suffering from chronic respiratory disease, such as cystic fibrosis, primary ciliary } \\ & \text { dyskinesia or anatomical abnormalities. } \\ & \text { Only experiencing recurrent AOM or chronic suppurative otitis media without other } \\ & \text { recurrent RTIs. } \\ & \text { Known allergy to co-trimoxazole. } \\ & \text { Known contraindication for co-trimoxazole, for example, liver failure or impaired } \\ & \text { kidney function and/or haematologic disorders. }\end{array}$

${ }^{*}$ Recurrent upper RTIs: for children aged <2 years yearly at least 11 and for children aged 2-10 years yearly at least 8 parental-reported upper RTIs possibly including, but not limited to, otitis media. Recurrent lower RTIs (ie, pneumonia, bronchopneumonia or acute bronchitis) are defined as at least two episodes per year or three or more episodes during the child's life regardless of age..$^{34} 35$

AOM, acute otitis media.

2. To examine predictors (eg, demographic, environmental, family history, mucosal, microbiological and immunological characteristics) for the (absence of) prophylactic treatment effect.

3. To examine whether cessation of antibiotic prophylactic treatment affects the presence of RTI symptoms and how this correlates with clinical, microbiological and immunological characteristics of the patients.

4. To record and evaluate adverse events.

- The occurrence of mild adverse effects as described in the Summary of Product Characteristics, such as skin rash, gastrointestinal complaints, pruritus or mild headache.

- The occurrence of severe adverse reactions

5. To examine short-term and long-term effects of cotrimoxazole prophylaxis on microbiota deviation, AMR and (mucosal and systemic) immunological outcomes.

\section{Study design}

We will conduct a randomised placebo-controlled double-blind clinical trial in which we compare co-trimoxazole with a placebo in 158 children with recurrent upper and/or lower RTIs. The inclusion and exclusion criteria are listed in table 1 . We will enrol children aged 6 months up to 10 years. Children younger than 6 months will not participate in this study, because at this age, the presence of recurrent RTIs cannot be established yet. For age-specific definitions of recurrent RTIs, we took the twofold SD of the mean number of upper RTIs per year in a cohort of 1314 German children, except for children aged 5-10 years, in whom we used the same definition as younger children ( $2-5$ years). This means that we define recurrent RTIs as at least 11 parental-reported upper RTIs per year for children younger than 2 years and eight parental-reported upper RTIs per year for children aged 2-10 years. ${ }^{34}$ Recurrent lower RTIs (ie, pneumonia, bronchopneumonia or acute bronchitis) are defined as at least two episodes per year or three or more episodes during the child's life regardless of age. These definitions of recurrent upper and lower RTIs were also used in the Dutch guideline for diagnostic strategies in children with recurrent RTIs. ${ }^{35}$ If an underlying immune deficiency or contraindication for co-trimoxazole ${ }^{36}$ has not been ruled out yet, these will be tested in the blood sample taken in all participants before randomisation. Children will be randomised to one of two oral suspension regimens for 3 months: co-trimoxazole $36 \mathrm{mg} / \mathrm{kg} /$ day $(2 \times 18 \mathrm{mg} / \mathrm{kg})$ or placebo two times per day. The dose of co-trimoxazole is in accordance with the therapeutic dose (for acute infections) as described in the Dutch paediatric drug formulary. Doses and duration of treatment are also based on studies of antibiotic prophylaxis in paediatric populations with recurrent acute or chronic suppurative otitis media. ${ }^{15}$ In case of a new RTI episode occurring during follow-up for which the child has a clinical indication to receive (additional) antibiotic treatment, the child will receive antibiotics according to national guidelines and the study medication will be discontinued for the duration of this antibiotic course. Medication compliance will be measured in two ways. During the T3 visit to the hospital, parents will be asked to bring the bottles of trial medication and to answer questions about compliance. By doing so, we will be able to compare the self-reported compliance will the number of empty and (partly) full bottles that are returned.

\section{Randomisation}

The randomisation procedures are performed by a member of the trial pharmacy. Randomisation is computer-generated and subjects will be allocated in a 1:1 ratio with random block sizes of two, four or six subjects to prevent predictability of the allocation. Study medication 
Table 2 Schedule of enrolment, intervention and measurement of outcomes

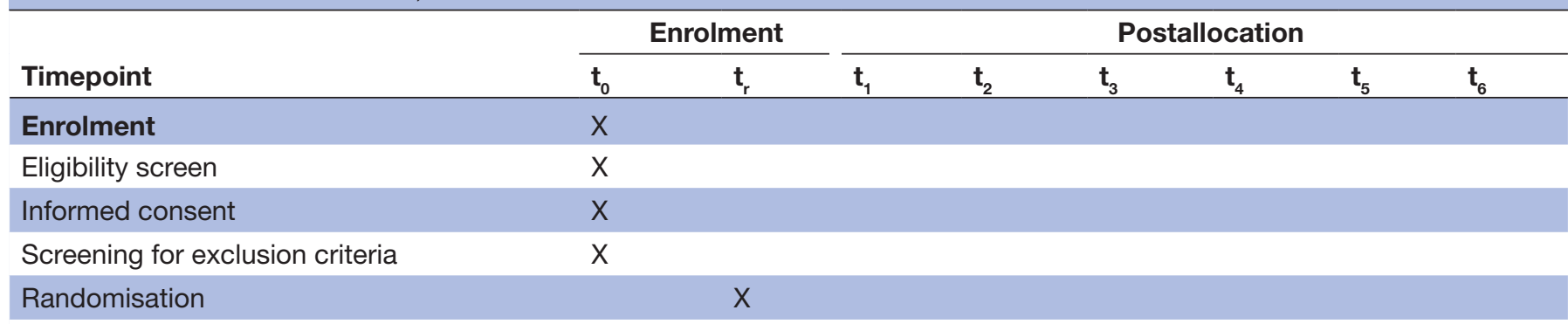

\section{Intervention}

Co-trimoxazole or placebo

\section{Measurements}

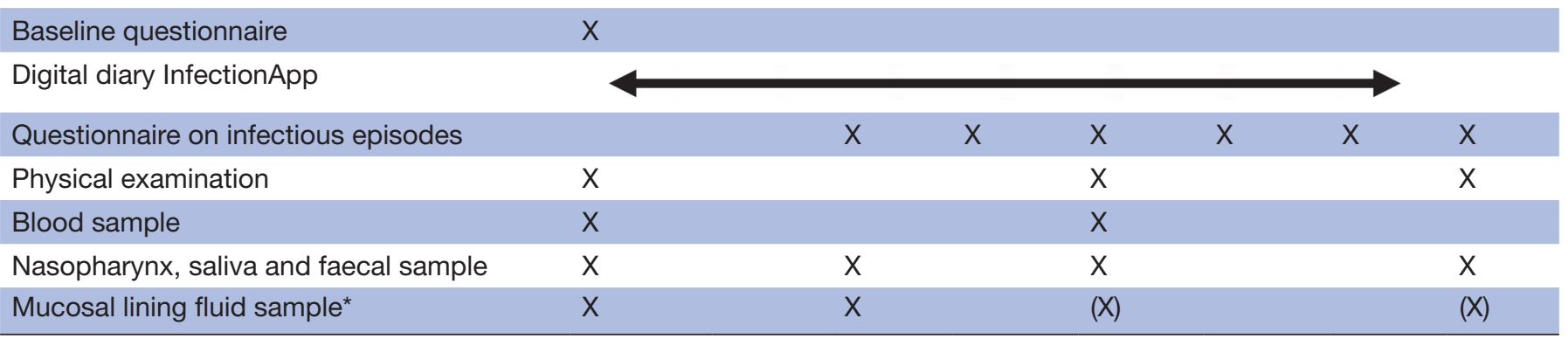

*Sampling at 2/4 time points, preferably T0 and T1.

is blinded for the subjects, his/her parent(s), physicians, the monitor and the study team. Only the members of the trial pharmacy have access to information on the allocation for each subject, because they are responsible for the preparation and delivery of the study medication, and for emergency de-blinding, if needed.

\section{Measurements}

The schedule of study enrolment, interventions and measurements of endpoints is shown in table 2. To record parent-reported data on symptoms of RTIs, a mobile application will be used. Previous studies used paper diaries to detect parent-reported symptoms. These are prone to non-compliance and may hamper real-time detection of disease occurrence. The use of our Diary-App for symptom recording costs parents less than 1 min per day and has shown to improve case-finding and questionnaire completeness from $60 \%$ to $\geq 90 \%$. $^{37}$ Also, parents will be asked to answer extra questions once a month. These include questions about the use of other antibiotics (see online supplemental file 1 for the monthly questionnaire).

At inclusion and after 1, 3 and 6 months $( \pm 2$ weeks for each sample point) non-invasive respiratory (nasopharyngeal swab and saliva) and faecal samples for microbiota composition, immunologic analysis and AMR gene detection will be collected. Also, these samples can be used for viral analysis. In addition, mucosal lining fluid will be collected at two time points in a subset of subjects. Before inclusion, blood samples will be taken to test for possible contraindications (eg, kidney and/or liver dysfunction). After treatment completion, blood samples will also be collected to monitor for possible kidney, liver and haematological side effects of co-trimoxazole use. Extra blood samples will be collected for additional immunological analyses at both time points. Before the start of treatment, parents will be asked to fill in a questionnaire. The study will be started in a minimum of 10 hospitals in the Netherlands. Inclusion will take place during both the winter and summer period to account for seasonal differences in microbiota composition in our analyses. If necessary, the number of study locations can be extended, depending on the speed of subject enrolment.

\section{Sample size}

We assumed $90 \%$ compliance with symptom monitoring via the app, that is, app data available for $0.90 \times 3$ months (90 days) $=81$ days per participant. There is limited literature available about the number of days with RTI symptoms per time period in children with recurrent RTIs. Toivonen et al published a large prospective cohort study from Finland including 1089 children followed up from birth to 2 years of age for respiratory infections by a daily symptom diary. ${ }^{1}$ In this study, children with recurrent RTIs (defined as number of days with symptoms $>90 \%$ percentile limit) had a median of 31 days per 100 days with at least one respiratory symptom. In a pilot study performed in the winter season in one of the participating centres (UMC Utrecht) including 18 children with recurrent RTIs, we observed a median of 76 days with at least one respiratory symptom and a median of 42 days with at least two respiratory symptoms per 100 days (this would be 34 per 81 days). We estimated that the median number of days with at least two symptoms in the Finnish study 
would be $(42 / 76) * 31=17$ days per $100=14$ days per 81 days. Following, we took the average of the Finnish study and our own study to end up with an estimated median number of symptomatic days of $(14+34) / 2=24$ days per 81 days in the placebo group.

The IQR in the Finnish study was 104-136, translating into a SD of 23.7, which was comparable to the SD in our pilot study (20.5); as such we took the SD of our study for our sample size calculation since the period in which symptoms were measured in the pilot study (mean 115 days) better reflected the follow-up period of the trial (90 days) than the period in which symptoms were measured in the Toivonen study (at least 1 year).

Assuming 24 days with at least two RTI symptoms in the placebo group and taking our aim to detect a clinically meaningful $40 \%$ reduction (ie, a reduction of $0.40 \times 24$ $=10$ days with RTI symptoms) in the antibiotic treatment arm, we need to include 71 children per arm, so a total number of $71 \times 2=142$ children, according to the Wilcoxon-Mann-Whitney test for two groups (two-tailed) with alfa 0.05 and power $80 \%$. Including a dropout rate of $10 \%$, this brings us to a number of $71 / 0.9=79$ children per arm, so a total number of 158 children.

\section{Outcomes and data analysis}

Table 2 summarises the assessment and sampling schedule.

\section{Assessment of RTI symptoms}

Descriptive statistics for demographic and clinical characteristics for both treatment allocation groups will be described. These include but are not limited to age, height, weight, ethnicity, co-morbidity, previous ENTinterventions, use of medication, the number of RTI episodes before inclusion, the severity of the infections (eg, hospital admissions) and type of RTI (eg, bronchiolitis, otitis, pneumonia). Also, we will examine the frequency distribution of risk factors for the development of RTIs, which include for example smoking in the household, number of siblings and daycare attendance.

Analyses will be performed on the basis of the 'intentionto-treat principle', comparing the treatment arm with the placebo arm, defining the treatment group based on the treatment allocation. In the intention-to-treat analysis, every randomised subject will be included according to treatment assignment, thus ignoring potential nonadherence, protocol deviations, withdrawal and anything that happens after randomisation, therefore maintaining prognostic balance generated from the original random treatment allocation. The analyses will only include subjects of whom at least $80 \%$ of the symptom diary data is available. An inventory of missing data will be made and if over $5 \%$ of 142 subjects have less available data than $80 \%$ of the symptom diary days, an imputation strategy will be used. We will also conduct a 'per-protocol analysis' in which we will only include the days that patients adhered to the protocolled treatment allocation. $\mathrm{R}$ and IBM SPSS Statistics will be used for statistical analyses. ${ }^{38}$
For our primary objective, we will compare the number of days with at least two RTI symptoms during 90 days of receiving antibiotic treatment/placebo. Since the actual number of monitored days may vary per patient, we will analyse the incidence rate (number of days with at least two RTI symptoms divided by the total number of days monitored). We will use a negative binomial regression analysis with the number of days with at least two respiratory symptoms as outcome and the number of days monitored as offset. Our target parameter is the incidence rate ratio for treatment. We will include main effects of strong predictors of RTI symptoms in the model.

To determine which set of available determinants predicts heterogeneity of treatment effect, we will first develop a prediction model including well-known risk factors for the development of RTI complaints in children. These include, for example, age of the child, smoke exposure in the household, day-care attendance and number of siblings. In this prediction model, we will adjust for treatment allocation. ${ }^{39}$ From this model, a summary score will be derived that is used as a risk score in the final model. The interaction between this risk score and the treatment allocation will be added as a covariate in a model for the primary outcome to investigate to what extent these host factors affect the effect of treatment on our primary outcome.

As a secondary analysis, we will also perform a negative binominal regression analysis with number of days with at least two RTI symptoms as the dependent variable and number of monitored days as offset, during the total 0-6 months period in order to assess the outcome both during and after cessation of co-trimoxazole versus placebo. In addition, we will also apply a mixed-effect model to estimate whether the pattern of RTI symptoms over time changes according to treatment allocation.

\section{Respiratory and gut microbiota}

For the secondary objective to detect shifts in microbiota composition in the group that received antibiotic prophylaxis compared with the placebo group, we will collect nasopharyngeal swabs (paediatric Copan e-swab with flocked nylon tip) and faecal samples from which bacterial DNA will be extracted according to previously validated methods. Swabs and faecal samples are frozen at $-80^{\circ} \mathrm{C}$ until further analyses. Metagenomic sequencing will be conducted in order to identify the microbiota composition and AMR genes of the faecal samples, in addition to antimicrobial resistance analyses of specific bacteria. For nasopharyngeal samples, 16S-based sequencing will be used to examine the microbiota composition and follow-up analyses for AMR genes or resistance analyses for specific bacteria may take place..$^{30} 40$

\section{Saliva, blood and mucosal lining fluid samples}

Blood samples (peripheral blood mononuclear cells and plasma), saliva samples and mucosal lining fluid will be collected at predetermined intervals (table 2) for immunological analyses aimed at the identification of markers 
associated with clinical outcome and treatment effect, and further exploration of the immune response against RTIs/pathogens and microbiota-immune interactions. Techniques include, but are not limited to, flow cytometry, proteomics, proliferative studies, cytokine release assays and RNA expression profiling. Saliva samples will also be used for the determination of antimicrobial peptides, inflammatory cytokines, proteomics and mucosal antibodies. Mucosal lining fluid will be used to measure (protein) immune markers such as antibodies, chemokines and cytokines. Finally, saliva/MLF/nasopharyngeal swab samples can be used for viral analyses.

\section{Data management}

Clinical data will be collected from the Electronic Medical Record by the research staff. All research data will be stored in the data management programme Castor Electronic Data Capture (EDC). The handling of personal data will comply with the Dutch General Data Protection Regulation (in Dutch: Algemene Verordening Gegevensbescherming). Data will be handled confidentially. The research team has access to coded data. To be able to trace data to an individual subject, a subject identification code list will be used to link the research data to the subject, which will be safeguarded by the local investigators and the trial pharmacy. This trial is monitored in accordance with the Good Clinical Practice guidelines.

\section{PATIENT AND PUBLIC INVOLVEMENT}

Because we are supported by two patient involvement groups, we feel confident that both physicians and also patients will be open to our study results. The patient involvement groups were actively involved in the development of our study protocol. During the yearly conference day of the Foundation for Primary Immune Deficiencies ('Stichting voor Afweerstoornissen'), one of our group members (LMV) has discussed the use of antibiotic prophylactic treatment for mild immune deficiencies in children with patients and parents. Many patients and parents expressed their worries about prolonged antibiotic use, mainly related to its adverse effects and the possibility of future infections with resistant bacteria that can no longer be treated with antibiotics. Following this discussion, we started discussion sessions with parents of children visiting the airway clinic in the Wilhelmina Children's Hospital, UMC Utrecht, at several time points. The results showed that parents felt that more research was needed to determine whether antibiotic prophylaxis is an effective treatment. The involvement of both patient groups facilitates acceptance and implementation of our study results in the patient community. We will provide these patient support groups with our study results by publication of the outcomes in 'Paraplu', the monthly journal of the Foundation for Primary Immune Deficiencies, and by discussion of the results during meetings of the patient support groups. Also, we will send a half-yearly newsletter and an information bulletin containing the final results to the study participants.

\section{ETHICS AND DISSEMINATION}

This study will be conducted according to the principles of the Declaration of Helsinki (2013, Brazil, version 64) and in accordance with the Research Involving Human Subjects Act $(W M O)$. The Medical Research Ethics committee Zuidwest Holland/LDD (The Hague/Leiden, The Netherlands) has approved the study protocol. Approval of the local board of each trial site will be obtained before enrolment of the first subject in that specific hospital. This study is registered in The Netherlands National Trial Register (NTR) as Trial NL7044. After completion of this study, results will be submitted to a peer-reviewed journal.

\section{CURRENT TRIAL STATUS}

The first subject was enrolled in January 2019. All the local boards of the first 10 trial sites have given their approval to start enrolling patients in this study, the latest approval was obtained in February 2020. If enrolment is slower than expected, a request for the addition of extra trial sites will be submitted to the Medical Research Ethics committee.

\section{Author affiliations}

${ }^{1}$ Department of Paediatrics, Haga Hospital, Juliana Childrens Hospital, Den Haag, Zuid-Holland, The Netherlands

${ }^{2}$ Department of Biomedical Data Sciences, Medical Statistics, Leiden University Medical Center, Leiden, The Netherlands

${ }^{3}$ Department of Hospital Pharmacy, Haga Teaching Hospital, Den Haag, The Netherlands

${ }^{4}$ Department of Hospital Pharmacy and Department of Epidemiology, Erasmus MC, Rotterdam, The Netherlands

${ }^{5}$ Center for Inflammation Research, Queen Medical Research Institute, University of Edinburgh, Edinburgh, UK

${ }^{6}$ Department of Paediatric Infectious Diseases and Immunology, University Medical Center Utrecht, Wilhelmina Children's Hospital, Utrecht, The Netherlands

${ }^{7}$ Department of Paediatrics, Erasmus MC Sophia, Rotterdam, The Netherlands ${ }^{8}$ Department of Paediatrics, Maastricht UMC+, Maastricht, The Netherlands

${ }^{9}$ Section Paediatric Infectious Diseases, Laboratory of Medical Immunology, Radboud Center for Infectious Diseases, Radboud Institute for Molecular Life Sciences, Nijmegen, The Netherlands

${ }^{10}$ Department of Paediatric Infectious Diseases and Immunology, Radboud University Medical Center, Amalia Children's Hospital, Nijmegen, The Netherlands

Contributors All authors helped with the design of this study and approved the final manuscript. DP, LMV and GJAD wrote the first draft of the study protocol and manuscript. NvG was consulted for the statistical analysis plan for this study. LEV helped in preparing the trial medication and advised on improving safety measures in relation to the trial medication. DB was consulted for the secondary outcomes on microbiota composition and AMR genes. AMvR was consulted for the design of the study.

Funding This study was supported by the Elisabeth von Freyburg fonds, 'Stichting Kiddy Goodpills' and the Haga Teaching Hospital. Award/Grant number is not applicable.

Competing interests None declared.

Patient consent for publication Not required.

Provenance and peer review Not commissioned; externally peer reviewed.

Supplemental material This content has been supplied by the author(s). It has not been vetted by BMJ Publishing Group Limited (BMJ) and may not have been 
peer-reviewed. Any opinions or recommendations discussed are solely those of the author(s) and are not endorsed by BMJ. BMJ disclaims all liability and responsibility arising from any reliance placed on the content. Where the content includes any translated material, BMJ does not warrant the accuracy and reliability of the translations (including but not limited to local regulations, clinical guidelines, terminology, drug names and drug dosages), and is not responsible for any error and/or omissions arising from translation and adaptation or otherwise.

Open access This is an open access article distributed in accordance with the Creative Commons Attribution Non Commercial (CC BY-NC 4.0) license, which permits others to distribute, remix, adapt, build upon this work non-commercially, and license their derivative works on different terms, provided the original work is properly cited, appropriate credit is given, any changes made indicated, and the use is non-commercial. See: http://creativecommons.org/licenses/by-nc/4.0/.

\section{ORCID iDs}

Daphne Peeters http://orcid.org/0000-0002-3110-9434

Lilly M Verhagen http://orcid.org/0000-0002-3451-9201

\section{REFERENCES}

1 Toivonen L, Karppinen S, Schuez-Havupalo L, et al. Burden of recurrent respiratory tract infections in children: a prospective cohort study. Pediatr Infect Dis J 2016;35:e362-9.

2 Ball TM, Holberg CJ, Aldous MB, et al. Influence of attendance at day care on the common cold from birth through 13 years of age. Arch Pediatr Adolesc Med 2002;156:121-6.

3 Otters HBM, van der Wouden JC, Schellevis FG, et al. Changing morbidity patterns in children in Dutch general practice: 1987-2001. Eur J Gen Pract 2005;11:17-22.

4 Schaad UB, Esposito S, Razi CH. Diagnosis and management of recurrent respiratory tract infections in children: a practical guide. Arch Pediatr Infect Dis 2016;4:e31039.

5 Couriel J. Assessment of the child with recurrent chest infections. $\mathrm{Br}$ Med Bull 2002;61:115-32.

6 Murray MT, Heitkemper E, Jackson O, et al. Direct costs of acute respiratory infections in a pediatric long-term care facility. Influenza Other Respir Viruses 2016;10:34-6.

7 Enserink R, Lugnér A, Suijkerbuijk A, et al. Gastrointestinal and respiratory illness in children that do and do not attend child day care centers: a cost-of-illness study. PLoS One 2014;9:e104940.

8 Edmond K, Scott S, Korczak V, et al. Long term sequelae from childhood pneumonia; systematic review and meta-analysis. PLoS One 2012; 7:e31239.

9 Brower KS, Del Vecchio MT, Aronoff SC. The etiologies of non-CF bronchiectasis in childhood: a systematic review of 989 subjects. BMC Pediatr 2014;14:4

10 Thomson M, Myer L, Zar HJ. The impact of pneumonia on development of chronic respiratory illness in childhood. Pediatr Allergy Immunol Pulmonol 2010;23:279-90.

11 Onakpoya IJ, Hayward G, Heneghan CJ. Antibiotics for preventing lower respiratory tract infections in high-risk children aged 12 years and under. Cochrane Database Syst Rev 2015;9:CD011530.

12 Esposito S, Bianchini S, Bosis S, et al. A randomized, placebocontrolled, double-blinded, single-centre, phase IV trial to assess the efficacy and safety of OM-85 in children suffering from recurrent respiratory tract infections. J Trans/ Med 2019;17:284.

13 Esposito S, Marchisio P, Cavagna R, et al. Effectiveness of influenza vaccination of children with recurrent respiratory tract infections in reducing respiratory-related morbidity within the households. Vaccine 2003;21:3162-8.

14 Bugova G, Janickova M, Uhliarova B, et al. The effect of passive smoking on bacterial colonisation of the upper airways and selected laboratory parameters in children. Acta Otorhinolaryngol Ital 2018;38:431-8.

15 Leach AJ, Morris PS. Antibiotics for the prevention of acute and chronic suppurative otitis media in children. Cochrane Database Syst Rev 2006;4:CD004401.

16 Chintu C, Bhat GJ, Walker AS, et al. Co-trimoxazole as prophylaxis against opportunistic infections in HIV-infected Zambian children (CHAP): a double-blind randomised placebo-controlled trial. Lancet 2004;364:1865-71.
17 Hughes WT, Kuhn S, Chaudhary S, et al. Successful chemoprophylaxis for Pneumocystis carinii pneumonitis. $N$ Engl J Med 1977;297:1419-26.

18 Genel F, Kutukculer N, Prospective KN. Prospective, randomized comparison of OM-85 bv and a prophylactic antibiotic in children with recurrent infections and immunoglobulin $A$ and/or $G$ subclass deficiency. Curr Ther Res Clin Exp 2003;64:600-15.

19 Rankine-Mullings AE, Owusu-Ofori S. Prophylactic antibiotics for preventing pneumococcal infection in children with sickle cell disease. Cochrane Database Syst Rev 2017;10:CD003427.

20 Saiman L, Anstead M, Mayer-Hamblett N, et al. Effect of azithromycin on pulmonary function in patients with cystic fibrosis uninfected with Pseudomonas aeruginosa: a randomized controlled trial. JAMA 2010;303:1707-15.

21 Clement A, Tamalet A, Leroux E, et al. Long term effects of azithromycin in patients with cystic fibrosis: a double blind, placebo controlled trial. Thorax 2006;61:895-902.

22 Church JA, Fitzgerald F, Walker AS, et al. The expanding role of co-trimoxazole in developing countries. Lancet Infect Dis 2015;15:327-39.

23 Ghilchik MW, Morris AS, Reeves DS. Immunosuppressive powers of the antibacterial agent trimethoprim. Nature 1970;227:393-4.

24 Rozin A, Schapira D, Braun-Moscovici Y, et al. Cotrimoxazole treatment for rheumatoid arthritis. Semin Arthritis Rheum 2001;31:133-41.

25 Emmanouilides CE, Lianou PE, Bassaris HP, et al. Trimethoprim, sulphamethoxazole, bacterial adhesion and polymorphonuclear leucocyte function. J Antimicrob Chemother 1990;26:803-12.

26 Wolf $\mathrm{J}$. Antibiotic resistance threatens the efficacy of prophylaxis. Lancet Infect Dis 2015;15:1368-9.

27 Pettigrew MM, Johnson JK, Harris AD. The human microbiota: novel targets for hospital-acquired infections and antibiotic resistance. Ann Epidemiol 2016;26:342-7.

28 Bedford Russell AR, Murch SH. Could peripartum antibiotics have delayed health consequences for the infant? BJOG 2006;113:758-65.

29 Moore AM, Patel S, Forsberg KJ, et al. Pediatric fecal microbiota harbor diverse and novel antibiotic resistance genes. PLoS One 2013;8:e78822.

30 Reyman M, van Houten MA, van Baarle D, et al. Impact of delivery mode-associated gut microbiota dynamics on health in the first year of life. Nat Commun 2019;10:4997.

31 Sommer MOA, Dantas G. Antibiotics and the resistant microbiome. Curr Opin Microbiol 2011;14:556-63.

32 Bogaert D, De Groot R, Hermans PWM. Streptococcus pneumoniae colonisation: the key to pneumococcal disease. Lancet Infect Dis 2004;4:144-54

33 Biesbroek G, Sanders EAM, Roeselers G, et al. Deep sequencing analyses of low density microbial communities: working at the boundary of accurate microbiota detection. PLoS One 2012;7:e32942

34 Grüber C, Keil T, Kulig M, et al. History of respiratory infections in the first $12 \mathrm{yr}$ among children from a birth cohort. Pediatr Allergy Immunol 2008;19:505-12.

35 ErasmusMC-Sophia in collaboration with the pulmonary, infectious diseases and immunology section of the Dutch Paediatric Society, Dutch College of General Practioners (NHG) and 'Stichting kind en ziekenhuis'. (2016). Evidence-based richtlijn diagnostiek naar onderliggende aandoeningen bij kinderen met recidiverende luchtweginfecties. Available: https://www.kinderinfectieziekten.nl/

36 Summary of product characteristics (SMPC) CO-TRIMOXAZOL, medicines evaluation board (MEB) the Netherlands, RVG 10550 SPC 0817.23v.RS.

37 Prins-van Ginkel AC, de Hoog MLA, Uiterwaal C, et al. Detecting acute otitis media symptom episodes using a mobile APP: cohort study. JMIR Mhealth Uhealth 2017;5:e181.

38 R Core Team. R: a language and environment for statistical computing. Vienna, Austria: R Foundation for Statistical Computing, 2019. https://www.R-project.org/

39 Kent DM, Paulus JK, van Klaveren D, et al. The predictive approaches to treatment effect heterogeneity (path) statement. Ann Intern Med 2020;172:35-45.

40 Man WH, van Houten MA, Mérelle ME, et al. Bacterial and viral respiratory tract microbiota and host characteristics in children with lower respiratory tract infections: a matched case-control study. Lancet Respir Med 2019;7:417-26. 